\title{
Simulation of a 6 MV Elekta Precise Linac photon beam using GATE/GEANT4
}

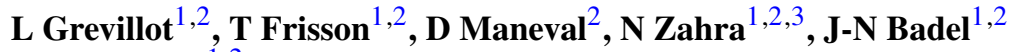 \\ and D Sarrut ${ }^{1,2}$ \\ ${ }^{1}$ Université de Lyon, Creatis CNRS UMR 5220, INSA, F-69622 Lyon, France \\ ${ }^{2}$ Centre de Lutte Contre le Cancer Léon Bérard, F-69373, Lyon, France \\ 3 IPNL, CNRS UMR 5822, F-69622, Villeurbanne, France \\ E-mail: loic.grevillot@gmail.com
}

Received 16 July 2009, in final form 19 September 2010

Published 19 January 2011

Online at stacks.iop.org/PMB/56/903

\begin{abstract}
The GEANT4-based GATE Monte Carlo (MC) platform was initially focused on PET and SPECT simulations. The new release v6.0 (February 2010) proposes new tools dedicated for radiation therapy simulations. In this work, we investigated some part of this extension and proposed a general methodology for Linac simulations. Details of the modeling of a $6 \mathrm{MV}$ photon beam delivered by an Elekta Precise Linac, with radiation fields ranging from $5 \times 5$ to $30 \times 30 \mathrm{~cm}^{2}$ at the isocenter are presented. Comparisons were performed with measurements in water. The simulations were performed in two stages: first, the patient-independent part was simulated and a phase space $(\mathrm{PhS})$ was built above the secondary collimator. Then, a multiple source model (MSM) derived from the $\mathrm{PhS}$ was proposed to simulate the photon fluence interacting with the patient-dependent part. The selective bremsstrahlung splitting (SBS) variance reduction technique proposed in GATE was used in order to speed up the accelerator head simulation. Further investigations showed that the SBS can be safely used without biasing the simulations. Additional comparisons with full simulations performed on the EGEE grid, in a single stage from the electron source to the water phantom, allowed the evaluation of the MSM. The proposed MSM allowed for calculating depth dose and transverse profiles in 48 hours on a single $2.8 \mathrm{GHz}$ CPU, with a statistical uncertainty of $0.8 \%$ for a $10 \times$ $10 \mathrm{~cm}^{2}$ radiation field, using voxels of $5 \times 5 \times 5 \mathrm{~mm}^{3}$. Good agreement between simulations and measurements in water was observed, with dose differences of about $1 \%$ and $2 \%$ for depth doses and dose profiles, respectively. Additional gamma index comparisons were performed; more than $90 \%$ of the points for all simulations passed the $3 \% / 3 \mathrm{~mm}$ gamma criterion. To our knowledge, this feasibility study is the first one illustrating the potential of GATE for external radiotherapy applications.
\end{abstract}

(Some figures in this article are in colour only in the electronic version) 


\section{Introduction}

For many years, the OpenGATE collaboration ${ }^{4}$ has developed an open source software package for nuclear medicine simulations, with a focus on PET and SPECT imaging (Jan et al 2004). The new GATE 5 release v6.0 (February 2010) proposes new tools, extending the GATE possibilities toward radiation therapy simulations (Jan et al 2010). This platform, based on the widespread GEANT4 MC toolkit (Agostinelli et al 2003, Allison et al 2006) is a collaborative development by researchers from several international institutions. It proposes an open source platform allowing developers to create a simulation on the basis of simple macro-commands instead of handling tedious $\mathrm{C}++$ syntaxes. This paradigm ensures a quicker learning phase for novices and makes GATE macro files easy to share within the scientific community. The use of GATE has been well validated for PET/SPECT simulations (De Beenhouwer, 2009, Schmidtlein et al 2006). It has also been used for brachytherapy (Thiam et al 2008) and micro-CT applications (Brunner et al 2009). It still relies on GEANT4 validations for radiotherapy applications (Rodrigues et al 2004, Carrier et al 2004, Foppiano et al 2005, Poon and Verhaegen, 2005, Thiam et al 2008).

Other powerful Monte Carlo (MC) codes like EGSnrc/BEAMnrc have already been validated and optimized for radiotherapy (Kawrakow and Walters, 2006). Initially, the efforts of implementing a new GATE module for these applications were directed toward hadron therapy (Grevillot et al 2010, Zahra et al 2010, Frisson et al 2009), which is not compatible with BEAMnrc. Since the tools developed for hadron therapy applications are very similar to those required for conventional radiotherapy, a feasibility study to extend the new GATE module to conventional radiotherapy has been proposed. The different possibilities offered by GATE (Jan et al 2010) make it a unique and powerful MC platform for medical physics applications. Having a single code allowing the simulation of several specific applications (PET, SPECT, CT, internal/external radiotherapy, hadron therapy) should facilitate the use of $\mathrm{MC}$ in medical physics.

This paper intends to present a general methodology for linear accelerator (Linac) simulations using GATE v6.0 release (Jan et al 2010). The new set of tools specifically oriented toward radiation therapy simulations has been assessed by simulation of an Elekta Precise $6 \mathrm{MV}$ Linac with radiation fields ranging from $5 \times 5$ to $30 \times 30 \mathrm{~cm}^{2}$ at the isocenter. The possibility of extending GATE simulations toward complex IMRT treatments using CT data is described in Jan et al (2010). This study is available on the GATE website through the Advanced example section.

\section{Materials and methods}

\subsection{Reference data}

Reference measurements were performed in a $60 \times 60 \times 60 \mathrm{~cm}^{3}$ water tank, with a $100 \mathrm{~cm}$ source to surface distance (SSD). Percent depth doses (PDD) and profiles at 1.5, 5 and $10 \mathrm{~cm}$ depth in the $X$ (in-plane) and $Y$ (cross-plane) directions were measured for field sizes of $5 \times 5,10 \times 10,20 \times 20$ and $30 \times 30 \mathrm{~cm}^{2}$ at isocenter. They were determined based on monthly quality assurance (QA) measurements without additional smoothing. However, all depth-dose and dose profile measurements were normalized to the maximum dose during the QA process. Cross-plane profiles were available only for $10 \times 10$ and $20 \times 20 \mathrm{~cm}^{2}$ radiation fields. We used a Scanditronix Wellhofer CC13 ionization chamber having an active volume of $0.13 \mathrm{~cm}^{3}$

4 www.opengatecollaboration.org

5 GATE: GEANT4 Application for Emission Tomography. 
and the OmniPro acquisition and analysis software from IBA-Dosimetry. Measurements were performed with a millimetric step.

\subsection{Physics settings}

In GEANT4, electromagnetic interactions can be simulated using the standard or low-energy packages, with cross-sections defined from $1 \mathrm{keV}$ and $10 \mathrm{eV}$, respectively, depending on the processes (Geant4 2008). Differences between standard and low-energy models are not the subject of this work; detailed information can be found in the Geant 4 Physics Reference Manual (Geant4 2008). For radiotherapy simulations, similar results were obtained using both packages, but low-energy models were 4.5 and 2.2 times slower than standard models, for the patient-independent and the patient-dependent parts, respectively (Poon and Verhaegen, 2005). The patient-independent part corresponds to the accelerator head above the secondary collimator, while the patient-dependent part corresponds to the simulation of the photon interactions from the secondary collimator to the patient itself (figure 1). More recent studies tend to demonstrate that the low-energy package is better suited for low-energy photons (Faddegon et al 2008, Thiam et al 2008), while the standard package achieves better results for proton therapy applications (Jarlskog and Paganetti, 2008) and that the low-energy extension becomes important only at a $\mu \mathrm{m}$ scale. Thus, the standard package was selected for this feasibility study. The $\operatorname{dosel}^{6}$ (Sarrut and Guigues, 2008) size was set to $5 \times 5 \times$ $5 \mathrm{~mm}^{3}$, resulting in a sensitive volume of $0.125 \mathrm{~cm}^{3}$, which is close to the active volume of the ionization chamber. Based on these values, we selected production thresholds of $1 \mathrm{~mm}$ for electrons, positrons and photons ( $\frac{1}{5}$ of the voxel dimension). A $1 \mathrm{~mm}$ range cut in water corresponds to an energy cut of roughly $350 \mathrm{keV}$ for electrons and positrons, and $5 \mathrm{keV}$ for photons, which is compatible with both standard and low-energy packages. Default values were used for all other parameters. Further investigations on step size limitations and other GEANT4 parameters such as dRover or dRange (Apostolakis et al 2009) are also needed to assess their impact on dose calculation. The influence of relevant GEANT4 parameters were presented in Poon and Verhaegen (2005) for radiotherapy applications and in Grevillot et al (2010), Zahra et al (2010) for hadron therapy applications.

\subsection{Geometry}

Given the flexibility of GATE/GEANT4, the components of the Elekta Precise accelerator head were defined as precisely as possible, based on the manufacturer's specifications (figure 1) and assuming that the geometry, the medium compositions and the densities were exact.

\subsection{Electron source}

The electron source is determined by two main characteristics (Chetty et al 2007): the spot size and the mean energy. The mean energy influences both the depth-dose curve and the shape of the dose profiles, while the spot size has only an impact on the dose profiles. These parameters were adjusted according to the reference method described in Verhaegen and Seuntjens (2003). First, for adjusting the Gaussian electron beam mean energy, several simulations were performed with energy varying from 5 to $7 \mathrm{MeV}$, with increments of $0.1 \mathrm{MeV}$. The resulting dose distributions were compared to reference measurements (depth-dose and dose profiles). Increasing the mean energy shifted the build-up regions in depth and modified the profile flatnesses for the different depths.

6 dosel: dose scoring voxel. 


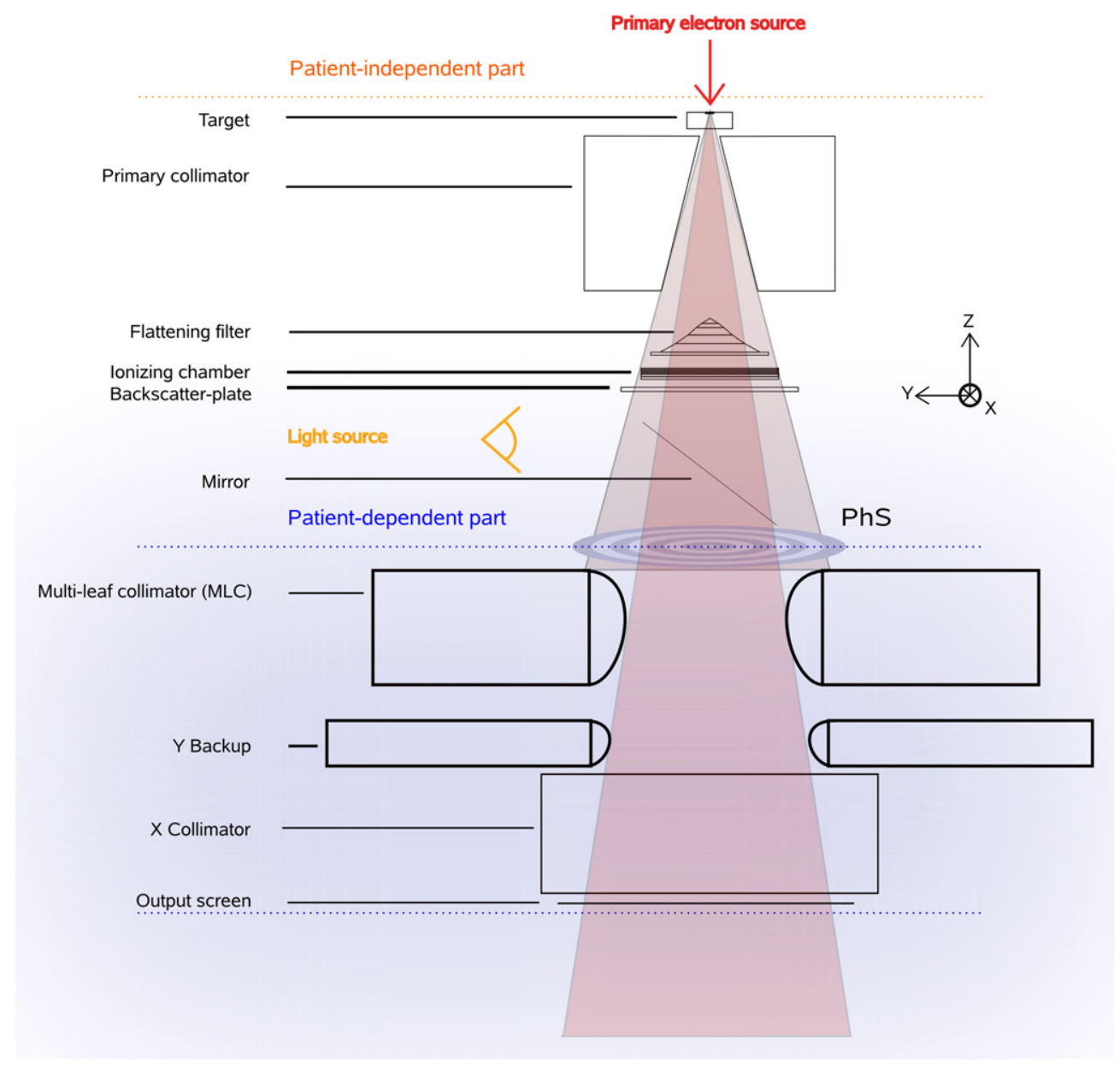

Figure 1. Description of the different geometrical elements of the accelerator head, including the location of the PhS above the secondary collimator.

We found that a mean electron beam energy of $5.8 \mathrm{MeV}$ best fits the measurements. After adjustment of the energy distribution, several simulations were performed with a FWHM Gaussian electron spot varying from 0 to $4 \mathrm{~mm}$ with a $1 \mathrm{~mm}$ step and were compared with the different dose profiles measured available. A FWHM electron spot of $3 \mathrm{~mm}$ was found to best fit the measurements. The spot size modifies the dose-gradient slope in the penumbra region and also the profile shape (profile flatness and 'horns' on the profile sides). The influence of different spot sizes on depth-dose simulation was verified. As recommended in the literature (Fix et al 2005), the FWHM energy was set to 3\% of the mean energy. The electron beam tuning stage was performed with the multiple source model (MSM) and then with full simulation on the $\mathrm{EGEE}^{7}$ grid (Camarasu-Pop et al 2010). Both led to the same electron beam parameters.

7 EGEE: Enabling Grids for E-sciencE project. 


\subsection{Error estimators}

Comparisons were performed in relative dose and not in absolute dose since all measurements were normalized to the maximum dose during the QA process. Simulated depth-doses were normalized to the integral dose and dose profiles were normalized to the mean dose in the profile center (within $2 / 3$ of the field size). Simulations were assessed by calculating the mean point-to-point dose error, with equation (1):

$$
\epsilon_{p}=\frac{1}{N} \sum_{i=1}^{N}\left(\frac{\left|d_{i}-\operatorname{dref}_{i}\right|}{\operatorname{dref}_{i}}\right),
$$

where $\epsilon_{p}$ is the mean point-to-point error, $i$ corresponds to a curve point index, $N$ is the number of points, $d_{i}$ is the dose computed at point $i$ and $d r e f_{i}$ is the reference dose measured at point $i$. Errors were also normalized to the maximum dose in order to increase the error weight at high doses and decrease it at lower values (equation (2)):

$$
\epsilon_{n}=\frac{1}{N} \sum_{i=1}^{N}\left(\frac{\left|d_{i}-\operatorname{dref}_{i}\right|}{\operatorname{dref}_{\max }}\right)
$$

where $d r e f_{\max }$ is the maximum dose measured. In high dose-gradient regions, large errors can occur, while the distance-to-agreement (DTA) can be small (Low et al 1998). Using $\epsilon_{p}$, point-to-point dose errors in low dose areas may lead to a high overall error. $\epsilon_{n}$ balance the point-to-point errors according to the dose deposited, so that the overall error calculated is more suited to characterize the simulation agreement with measurements. It was also found interesting to evaluate the simulations in terms of gamma index (Low et al 1998), taking into account both the maximum dose difference and the DTA requirements. We calculated the number of points passing the clinical $3 \% / 3 \mathrm{~mm}$ criterion. It means that we evaluated the dose difference between each simulated point of interest and the measured points within $3 \mathrm{~mm}$ around. The gamma index evaluation is presented in details in Low et al (1998). When $100 \%$ of the points pass the $3 \% / 3 \mathrm{~mm}$ comparison, it means that all points passed the comparison. The $3 \mathrm{~mm}$ criterion played a significant role in the high dose-gradient region, where large dose differences occur. The millimetric step of measurements allowed for evaluating the DTA with such a criterion. One has to be careful when analyzing the results since for small fields the number of points evaluated is very low (12 points only for a profile of a $5 \times 5 \mathrm{~cm}^{2}$ field). In this study, we aimed at evaluating the profile shapes. Points having a dose lower than $20 \%$ of the maximum dose (corresponding to the profile tails) were not taken into account for the comparisons. In the low dose areas, the statistical uncertainties increased due to the lack of primary event interactions and led to a nonrepresentative error increase. For depth doses, all points had a dose larger than $20 \%$ of the maximum dose, therefore all points were taken into account.

\subsection{Storing the fluence from the patient-independent section}

A phase space $(\mathrm{PhS})$ was used to store incoming particles before the secondary collimator (figure 1). The PhS was attached to a user-defined volume and stored the parameters of all the particles entering this volume for the first time (particle's type, 3D-coordinates, direction, energy, last interaction volume, production process, weight). In GATE, the $\mathrm{PhS}$ can be attached to any geometry in the simulation. We attached the PhS to a cylinder of $20 \mathrm{~cm}$ diameter and $1 \mathrm{~nm}$ thickness in the $z$ direction, so that all the particles stored were considered at the same $z$-position (figure 2). The $\mathrm{PhS}$ was located $1 \mathrm{~mm}$ above the secondary collimator and informations were stored as a ROOT file, which was processed using the ROOT software 


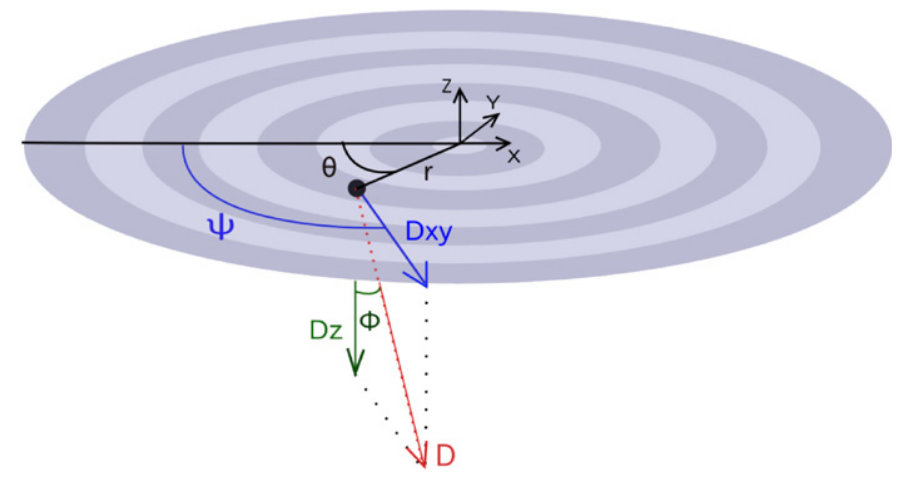

Figure 2. Particle coordinate system in the phase space. Photon position is defined by the angle $\theta$ and the radial distance $\mathrm{r}$. The photon direction is defined by the angles $\psi$ and $\phi . \vec{D}$ is the photon direction of length $\mathrm{D}$, composed of $\overrightarrow{D x y}$ : the azimuthal component in the xOy plan and $\overrightarrow{D z}$ : the vertical component.

(Rademakers and Brun, 1998). It is also possible to store PhS data in the IAEA format in order to interface GATE with other MC codes (Jan et al 2010). PhS storages between 2 and 170 million photons have been proposed (Schach von Wittenau et al 1999, Deng et al 2000, Fix et al 2005, 2004) depending mainly on the simulated field sizes. In accordance with the literature, we stored about 60 million photons, leading to a $\mathrm{PhS}$ of about $1.8 \mathrm{~GB}$. Additional tests using up to 10 times more photons did not show any differences in the results.

\subsection{Source model}

There are three different ways of simulating the dose deposition in the patient. One can either perform a full simulation using extensive computer resources like grid, or split the simulation into two parts and build a PhS file after the simulation of the patient-independent section. Then, to simulate the photon fluence interacting with the patient-dependent part, one can either read directly the PhS file or use a source model. The number of photons in a $\mathrm{PhS}$ file is fixed, hence the $\mathrm{PhS}$ undergoes statistical fluctuations known as 'latent variance' (Sempau et al 2001). Moreover, this requires to carry a bulky $\mathrm{PhS}$ of several GB for each simulation. In this study, a source model of the photon fluence in the accelerator was developed in order to smooth out possible fluctuations in the $\mathrm{PhS}$ file and also to propose a tool which does not require to carry such bulky PhSs. A source description file of $5 \mathrm{MB}$ and made of 5404 histograms was generated. This file represented the photon fluence in the accelerator head and was used as input by the MSM. The methodology allowing to sort out the PhS data into histograms is presented below.

Three major elements are known to influence the photon beam: the target, the primary collimator and the flattening filter (Schach von Wittenau et al 1999, Deng et al 2000, Fix et al 2004). Hence, the proposed photon fluence model derived from the $\mathrm{PhS}$ consisted of three sub-sources and was called MSM (Fix, 2001). The photon fluence in the accelerator head results from interactions between the primary electron source and the target, mainly via the bremsstrahlung process. Some high-energy photons can scatter in the geometry, while low energy ones are absorbed. Finally, the output beam consists of photons from the target, as well as secondary particles originating from the primary collimator, the flattening filter and the secondary collimator. We did not study the contribution of the secondary collimator, because 
(a)

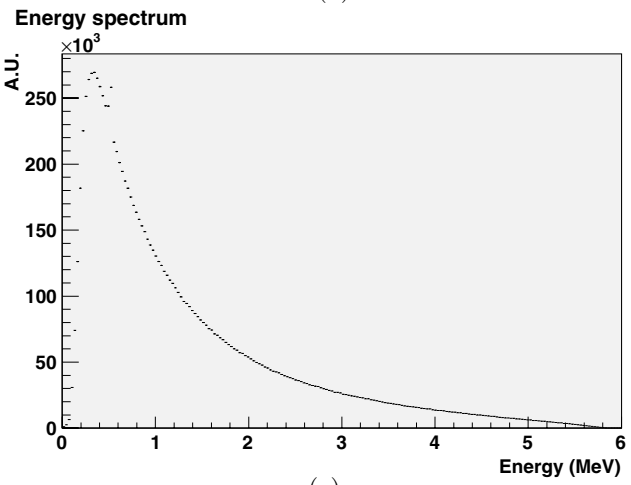

(c)

Energy fluence

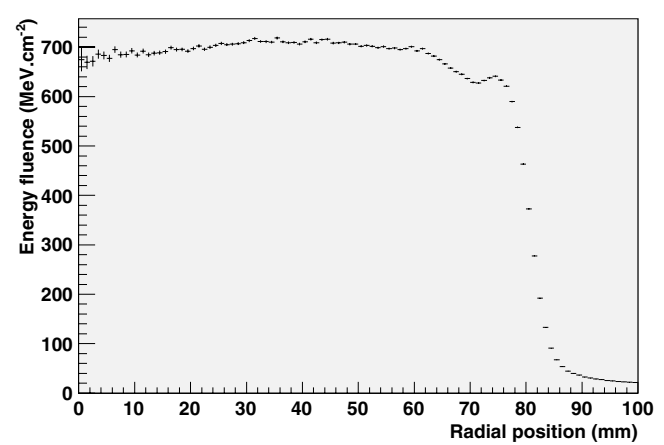

(b)

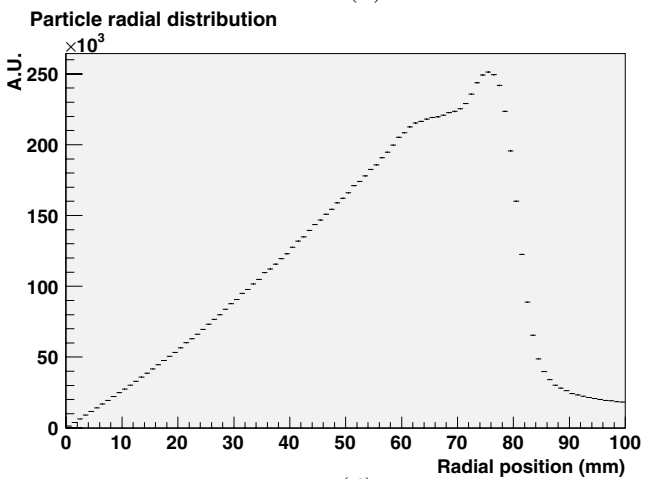

(d)

Radial mean energy

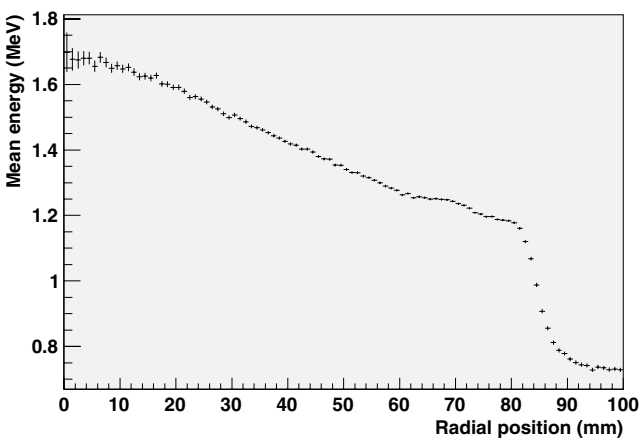

Figure 3. Major $\mathrm{PhS}$ parameters are presented and calculated using a $\mathrm{PhS}$ of $1.1 \times 10^{7}$ photons. (a) Energy spectrum of photons. (b) Photon radial distribution. (c) Photon energy fluence as a function of the radial position. (d) Photon mean energy as a function of the radial position. A step in the particle radial fluence is observed between 65 and $70 \mathrm{~mm}$ in (b), corresponding to interactions of the photons with the flat bottom edge of the flattening filter. After this plateau, a fluence increase is observed because the photons no longer interact with the flattening filter edge. Then, the fluence drops down due to the presence of the primary collimator in the photon path.

the MSM was located above. The major $\mathrm{PhS}$ parameters describing the photon fluence are presented in figures 3(a)-(d).

We defined the photon direction by two angles: $\psi$ and $\phi$ and the position with polar coordinates: the radial distance $r$ and the angle $\theta$ (figure 2). General distributions of $\psi$ and $\phi$ in the PhS are presented in figures 4(a) and 5(a). There is a known dependence between the photon energy and the distance to the center of the $\mathrm{PhS}$ (radius), with a mean energy increasing from the edge to the center (Schach von Wittenau et al 1999) (figure 3(d)). This correlation has been confirmed by several studies (Fix et al 2000, 2004, Chetty et al 2007, Cho et al 2005). We also found that the photon direction is linked both to energy and position (figures 4(b)-(d) and 5(b) and (c)).

As regards the photon position and energy, the $\mathrm{PhS}$ was divided in 200 radial bins (rings), with a constant radial step of $0.5 \mathrm{~mm}$. For each source (target, primary collimator, flattening filter), a 200 bins histogram was generated and contained the radial photon distribution. An energy histogram was associated with each ring and divided into 400 bins from 0 to $8 \mathrm{MeV}$, with an energy step of $20 \mathrm{keV}$. Because of the axial symmetry of the accelerator head, a uniform azimuthal photon distribution was assumed. 


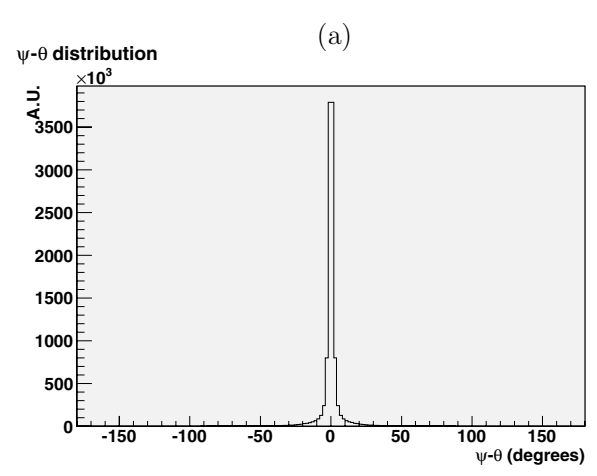

(c)

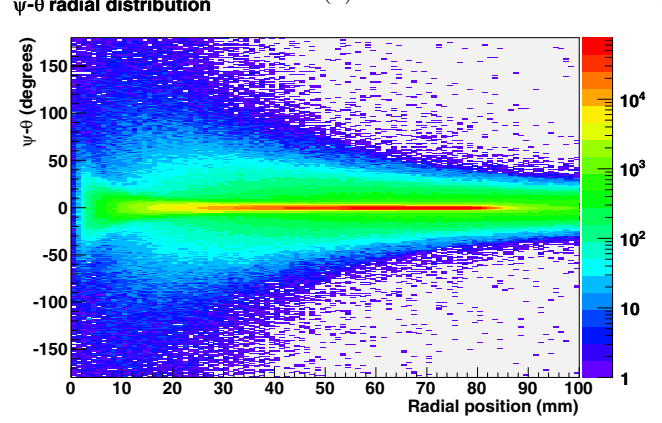

(b)

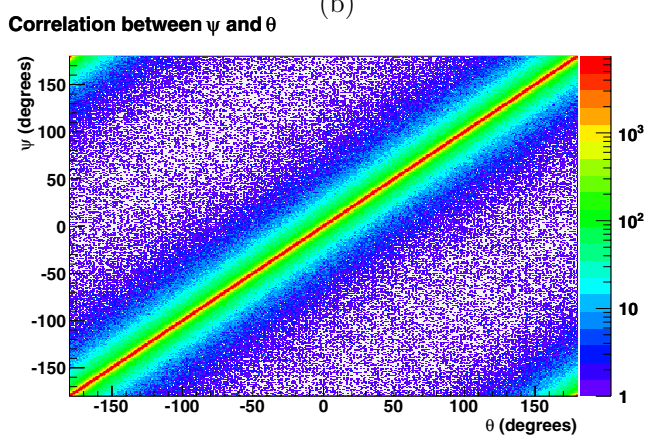

(d)

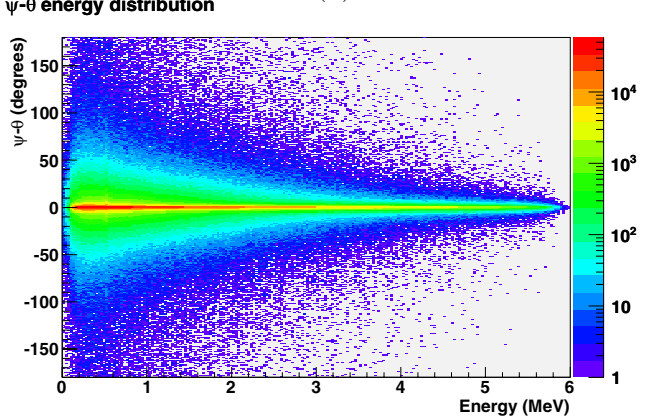

Figure 4. Analysis of the direction parameter $\psi$ using a $\mathrm{PhS}$ of $1.1 \times 10^{7}$ photons. Top: correlation between $\psi$ and $\theta(\mathrm{a}, \mathrm{b})$. Bottom: double dependence of $\psi$ with photon radial position (c) and energy (d).

As regards the photon direction, the $\mathrm{PhS}$ was divided in 40 radial bins, with a $2.5 \mathrm{~mm}$ step. A 20 bins energy histogram was associated with each ring, with a step of $400 \mathrm{keV}$. Each energy bin from each ring was associated with two histograms for the directions ( $\phi$ and $\psi)$. This double correlation between energy and radial position to select the photon direction was found to be mandatory in order to obtain a precise reproduction of the photon fluence. Because of this double correlation, the statistics in the direction histograms were relatively low, especially in the center of the PhS where few particles were stored (figure 3(b)), suggesting that larger bins or bigger $\mathrm{PhS}$ should be used. In order to account for the correlations between the different physical properties of the photons, small bin steps are required. However, the lower the bin steps, the higher the statistical uncertainty in each bin due to the lack of photons. The numbers of radial bins and energy bins were therefore determined empirically to achieve a balance between binning resolution and statistical uncertainty.

The MSM was studied for photons only. The electrons and positrons stored in the $\mathrm{PhS}$ were not taken into account in the simulations. They were neglected as they represented less than $0.5 \%$ of the total number of particles stored in the $\mathrm{PhS}$. It is noteworthy that the contaminant electrons produced by the secondary collimator were simulated, because the reconstructed photon source was located above. The MSM accuracy was assessed by comparing simulations with various depth doses and dose profiles measured in water (section 2.1). In this study, $\mathrm{PhS}$ files were generated using the selective bremsstrahlung splitting (SBS) tool in order to speed up the tuning stage of the electron beam, as described in section 2.8. Then, a reference $\mathrm{PhS}$ has been generated without the SBS tool. We will further refer to these $\mathrm{PhSs}$ as the reference 

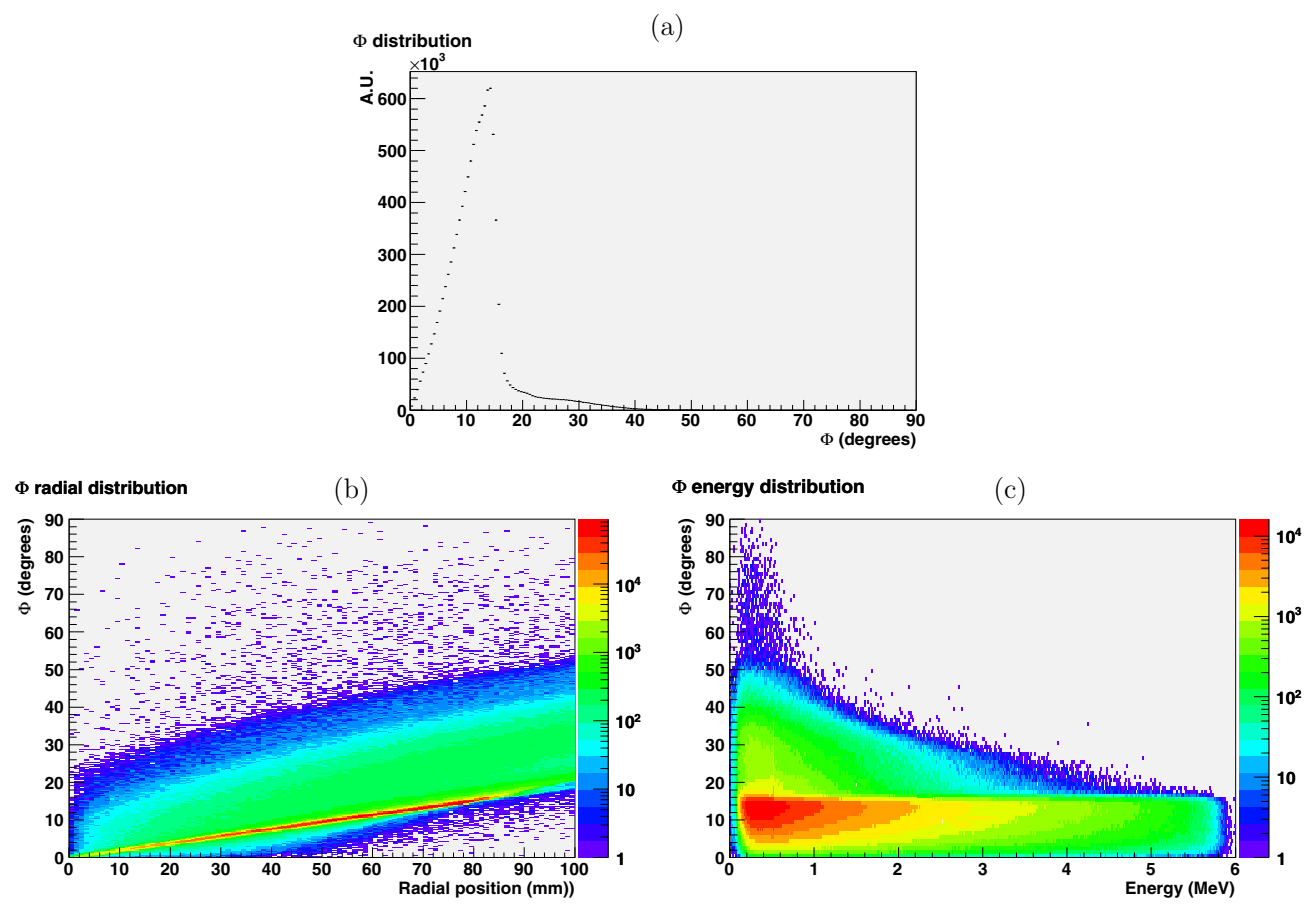

Figure 5. Analysis of the direction parameter $\phi$ using a PhS of $1.1 \times 10^{7}$ photons. Top: $\phi$ distribution (a). Bottom: double dependence of $\phi$ with photon radial position (b) and energy (c).

$\mathrm{PhS}$ and the SBS PhS. A further improvement in the toolkit would be to fill the histograms directly during the simulation, thus avoiding to store bulky $\mathrm{PhSs}$.

Depth doses and dose profiles were computed with the MSM and compared to the reference measurements, using both the reference and the SBS PhSs. These comparisons allowed for evaluating the bias introduced by the SBS tool when using the MSM. Additional calculations were performed using the EGEE grid for full simulations, from the electron source to the water phantom, using the SBS tool. These simulations allowed for evaluating the bias introduced by the MSM, when using the SBS tool. Eventually, some simulations were performed by reading the reference $\mathrm{PhS}$ and were compared to MSM calculations. It is noteworthy that it is possible to perform all kind of GATE simulations on the grid, for radiotherapy as well as for PET and SPECT applications. See Camarasu-Pop et al (2010) for implementation details of GATE on the EGEE grid.

\subsection{SBS tool}

The mandatory tuning stage of the two electron beam parameters (mean energy and spot size) required many simulations. A different $\mathrm{PhS}$ file corresponding to each configuration was used by the MSM in order to compare simulations with measurements in water. A variance reduction technique SBS (Rogers et al 2002), is now implemented in GATE (Jan et al 2010) in order to increase the production of photons by the bremsstrahlung process. The improvement of the simulation efficiency for radiotherapy applications is a complex task, which was extensively studied for the EGSnrc/BEAMnrc code: directional bremsstrahlung splitting (DBS) (Kawrakow et al 2004, Mainegra-Hing and Kawrakow, 2006), bremsstrahlung 
splitting number optimization (Kawrakow, 2005), bremsstrahlung cross-section enhancement (BCSE) (Ali and Rogers, 2007), particle recycling combined with photon splitting toward full Linac simulations (Kawrakow and Walters, 2006), etc.

In this study, we aimed at reducing the simulation time of the patient-independent section. Using the concept of filters described in Jan et al (2010), the SBS tool was selective in the direction; photons were produced only when the photon emission angle $\phi$ was lower than the direction criterion. We used a direction criterion of $20^{\circ}$, which was about three times the size of the primary collimator opening angle (of about $7^{\circ}$ ). When a primary electron hit the target and produce a bremsstrahlung photon, the SBS tool sampled $n$ photons at once, with a weight of $1 / \mathrm{n}$ each, all having the same energy and starting from the electron hit point in the target, only the photon direction was sampled, according to the direction criterion. User can define the splitting factor $n$, as well as the direction criterion. It is also possible to use other filters. Bremsstrahlung factors up to several hundreds have been reported (Verhaegen and Seuntjens, 2003). In this study, we applied a factor 100 . We compared the photon output rate with and without the SBS. The photon output rate was defined as the number of photons reaching a squared area of $60 \times 60 \mathrm{~cm}^{2}$ at a $100 \mathrm{~cm} \mathrm{SSD} \mathrm{for} \mathrm{a} \mathrm{fixed} \mathrm{number} \mathrm{of} \mathrm{primary} \mathrm{electrons.} \mathrm{We} \mathrm{also}$ characterized the SBS tool in terms of efficiency (Chetty et al 2007), taking into account the simulation time and the simulation statistical uncertainty, as proposed in Chetty et al (2006), using equation (3):

$$
\eta=\frac{1}{\left(\sigma_{D>0.5 D_{\max }}\right)^{2} \times T}
$$

where $\eta$ is the efficiency, $\sigma_{D>0.5 D_{\max }}$ is the simulation statistical uncertainty as defined in section 2.9 and $\mathrm{T}$ is the simulation time.

\subsection{Estimation of the dose calculation uncertainty}

A DoseActor tool (Jan et al 2010) was also used to score the dose deposition and its associated statistical uncertainty in the water phantom. In GATE, the dose/energy deposit is scored randomly along the step line by default, each hit being stored in the corresponding dosel. Advantages of this method are mentioned in Jan et al (2010). If the step is much longer than the dosel size, the energy will be deposited in one dosel selected randomly, which may affect the simulation efficiency. However, it is possible to set the maximum step length allowed using the stepLimiter process described in Jan et al (2010). It is noteworthy that each particle performs a step at geometrical boundaries. Concerning the variance reduction techniques, each particle is weighted as a function of its relative contribution. Such weights are used for dose deposit and uncertainty computation. The dose scored for a given particle interaction is equal to the energy released times the weight. The default particle weight is 1 and $1 / n$ for split photons and their daughter particles (photons, electrons, positrons), $n$ being the splitting factor. The statistical uncertainty in a dosel $i$ was estimated with the following formula (Walters et al 2002, Chetty et al 2007, 2006):

$$
\sigma_{i}=\sqrt{\frac{1}{N-1}\left(\frac{\sum_{j=1}^{N} X_{j}^{2}}{N}-\left(\frac{\sum_{j=1}^{N} X_{j}}{N}\right)^{2}\right)},
$$

where $\sigma_{i}$ is an estimate of the standard error of the mean dose in dosel $i, N$ is the number of primary independent histories and $X_{j}$ is the contribution to the scored quantity of an independent history $j$. In GATE, the recorded 'relative statistical uncertainty' corresponds to the ratio of $\sigma_{i}$ to the quantity scored in the dosel, so that the uncertainty is between 0 and 1 . The statistical 
Table 1. SBS photon output rate increase using the SBS tool, compared to a reference simulation performed without it.

\begin{tabular}{lllll}
\hline & Electrons & Time $(\mathrm{s})$ & Collected photons & Output rate $\left(\mathrm{s}^{-1}\right)$ \\
\hline Without SBS & $10^{7}$ & 2880 & 16464 & 5.7 \\
With SBS & $10^{7}$ & 35998 & 1997929 & 55.5 \\
\hline
\end{tabular}

uncertainty of the simulation was estimated using equation (5), as proposed in Chetty et al (2006):

$$
\sigma_{D>0.5 D_{\max }}=\sqrt{\frac{1}{K_{50}} \sum_{i=1}^{K_{50}}\left(\frac{\sigma_{i}}{D_{i}}\right)^{2}},
$$

where $\sigma_{D>0.5 D_{\max }}$ represents the simulation statistical uncertainty, $K_{50}$ the number of dosels receiving a dose higher than $50 \%$ of the maximal dose and $\left(\frac{\sigma_{i}}{D_{i}}\right)$ the relative statistical uncertainty.

In a previous study using GATE for brachytherapy applications, the global simulation uncertainty was assessed using recommendations of the AAPM Task Group $\mathrm{N}^{\circ} 43$ (Thiam et al 2008). In addition to the statistical uncertainty of the simulation, they took into account the geometrical and the cross-section uncertainties by quadratic summation. Details on cross-section evaluation in GEANT4 can be found in Poon and Verhaegen (2005). In a reference report written by the AAPM Task group $\mathrm{N}^{\circ} 105$ (Chetty et al 2007) for MC-based radiotherapy treatment planning, the photon cross-section uncertainty in the energy range of these applications was estimated between $1 \%$ and $2 \%$. Concerning the geometry, it is very difficult to determine a number representative of the geometrical uncertainty of the accelerator head. The measurement-based electron beam tuning stage has also a major influence. Therefore, there is no reference method proposed today for estimating the global uncertainty in radiotherapy simulations. Instead, various experimental verifications of MC algorithms are proposed in order to evaluate the simulations in heterogeneous media and to assess the photon beam model (Chetty et al 2007).

\section{Results and discussion}

\subsection{SBS assessment}

3.1.1. Photon output rate increase The photon output rate increase was estimated using the final electron beam parameters: electron beam mean energy $5.8 \mathrm{MeV}$, FWHM energy set to $3 \%$ and FWHM electron spot set to $3 \mathrm{~mm}$, with a SBS photon emission angle criterion of $20^{\circ}$. Results are summarized in table 1 . The photon output rate was increased by a factor of 9.7 $(55.5 / 5.7)$.

3.1.2. SBS efficiency The SBS efficiency was assessed by performing two full simulations, with and without SBS, from the electron source to the water tank. Each simulation included one depth dose and three dose profiles for a $10 \times 10 \mathrm{~cm}^{2}$ radiation field (as described in section 2.1). Results are given in table 2. Simulation efficiency was found to be $\frac{1.15 \times 10^{-1}}{1.85 \times 10^{-2}}=$ 6.2 times higher when using the SBS tool.

The efficiency calculation depends on both the photon output rate and the simulation of the photon interactions within the water phantom. The SBS tool allows for improving the 
Table 2. Estimated efficiencies with and without SBS.

\begin{tabular}{llll}
\hline & Time $(\min )$ & $\sigma_{D>0.5 D_{\max }}$ & $\eta$ \\
\hline Without SBS & 2170 & $1.58 \times 10^{-1}$ & $1.85 \times 10^{-2}$ \\
With SBS & 2170 & $6.32 \times 10^{-2}$ & $1.15 \times 10^{-1}$ \\
\hline
\end{tabular}

Table 3. Depth dose, in-plane and cross-plane profile evaluation for the MSM using the SBS PhS. Dose differences are evaluated using equations (1) and (2) and are given in percent. The percentage of points passing the $3 \% / 3 \mathrm{~mm}$ gamma comparison for the different curves is also presented.

\begin{tabular}{lllllllllll}
\hline Field size & Depth & \multicolumn{3}{c}{ In-plane } & \multicolumn{3}{c}{ Cross-plane } & \multicolumn{3}{c}{ Depth dose } \\
& & $\epsilon_{p}$ & $\epsilon_{n}$ & $\gamma$ & $\epsilon_{p}$ & $\epsilon_{n}$ & $\gamma$ & $\epsilon_{p}$ & $\epsilon_{n}$ & $\gamma$ \\
\hline \multirow{3}{*}{$5 \times 5 \mathrm{~cm}^{2}$} & $1.5 \mathrm{~cm}$ & 5.6 & 2.5 & 100 & - & - & - & & & \\
& $5 \mathrm{~cm}$ & 4.4 & 2.3 & 92 & - & - & - & 0.9 & 0.6 & 100 \\
& $10 \mathrm{~cm}$ & 3.9 & 2.3 & 100 & - & - & - & & & \\
& $1.5 \mathrm{~cm}$ & 3.6 & 2.0 & 100 & 3.9 & 2.2 & 95 & & \\
$10 \times 10 \mathrm{~cm}^{2}$ & $5 \mathrm{~cm}$ & 2.8 & 1.9 & 91 & 3.3 & 2.1 & 91 & 1.1 & 0.8 & 100 \\
& $10 \mathrm{~cm}$ & 4.2 & 1.9 & 100 & 3.6 & 1.7 & 96 & & \\
& $1.5 \mathrm{~cm}$ & 2.0 & 1.5 & 95 & 4.1 & 2.0 & 93 & & \\
$20 \times 20 \mathrm{~cm}^{2}$ & $5 \mathrm{~cm}$ & 1.9 & 1.2 & 95 & 3.2 & 1.7 & 98 & 0.7 & 0.5 & \\
& $10 \mathrm{~cm}$ & 2.1 & 1.4 & 98 & 3.6 & 1.7 & 91 & & \\
& $1.5 \mathrm{~cm}$ & 2.0 & 1.7 & 94 & - & - & - & & \\
$30 \times 30 \mathrm{~cm}^{2}$ & $5 \mathrm{~cm}$ & 2.1 & 1.6 & 95 & - & - & - & 1.1 & 0.8 & \\
& $10 \mathrm{~cm}$ & 2.8 & 2.0 & 93 & - & - & - & & & \\
\hline \multirow{2}{*}{30.5}
\end{tabular}

photon output rate, but does not influence the simulation within the water phantom itself. Therefore, increasing the photon output rate improves the global simulation efficiency, but with a lower factor: 6.2 instead of 9.7 in this case.

\subsection{Linac head modeling assessment}

It took less than one day to simulate the patient-independent part on a single $2.8 \mathrm{GHz}$ CPU with the SBS tool and one week without. PhS files of $1.8 \mathrm{~GB}$ were compressed into $5 \mathrm{MB}$ source description files containing 5404 representative histograms of the photon fluence in the Linac head. Regarding the patient-dependent section simulations, statistical uncertainties $\left(\sigma_{D>0.5 D_{\max }}\right)$ were below $3 \%$ for all simulations. For indicative purpose, the patient-dependent part simulation of a $10 \times 10 \mathrm{~cm}^{2}$ radiation field took approximately 2 days with one billion photons and the resulting simulation statistical uncertainty $\left(\sigma_{D>0.5 D_{\max }}\right)$ was $0.8 \%$. In comparison, full simulations performed on the grid last about 1 day in average. The main simulation macro file was automatically split and run through about 500 CPUs worldwide. The results were then automatically merged into a single output file. Equivalent simulations performed on a single $2.8 \mathrm{GHz}$ CPU would have last about 1 year. Results obtained with the MSM using the SBS PhS are summarized in table 3. One can see that depth doses were in very good agreement with measurements. The largest errors occurred for the profiles, with up to $5.6 \%$ discrepancy for $\epsilon_{p}$, for the $5 \times 5 \mathrm{~cm}^{2}$ radiation field. This is due to the fact that for small fields, few points were evaluated and the increased errors in the high dose-gradient regions significantly increased the overall error. Therefore, one can refer to $\epsilon_{n}$, which is more 

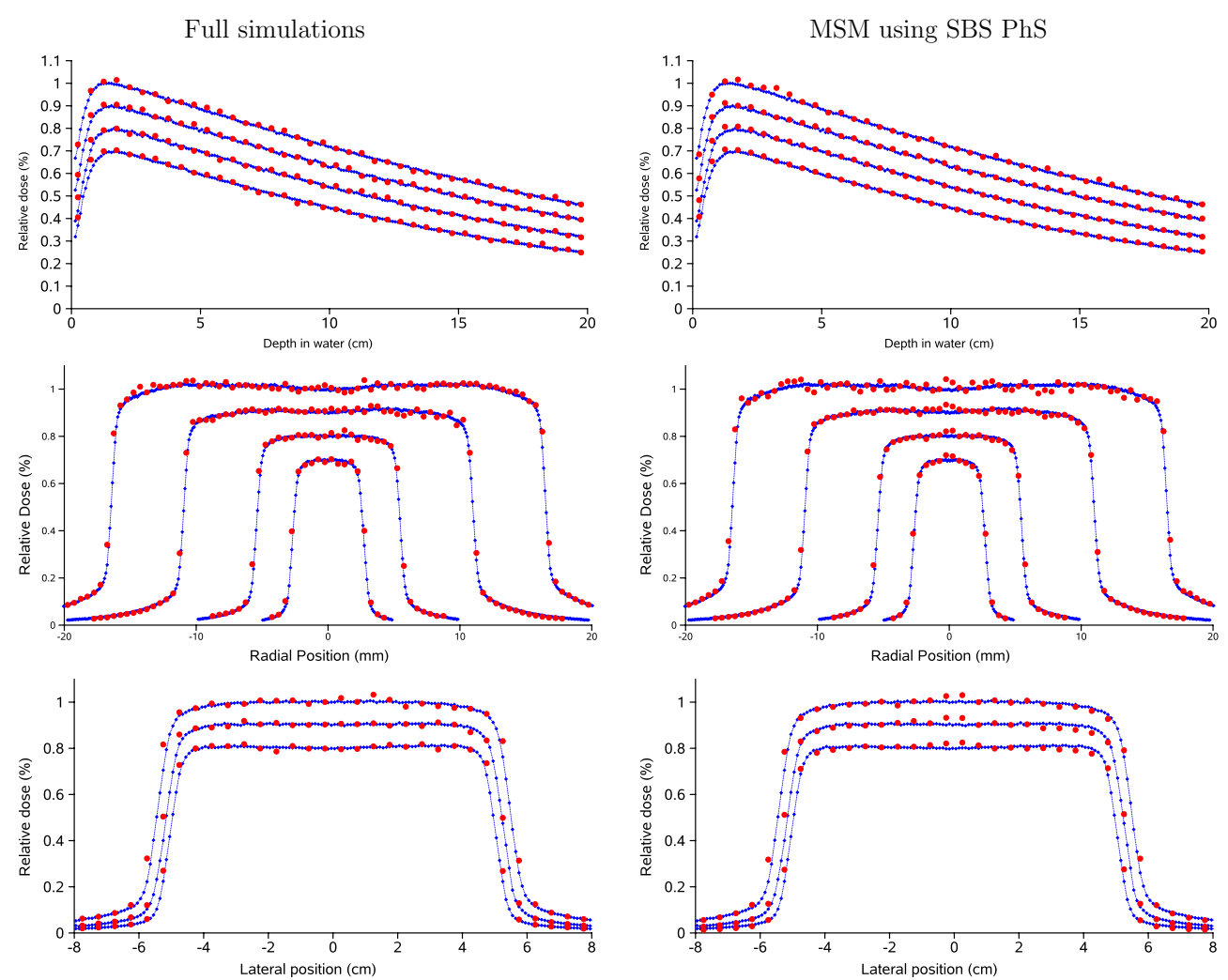

Figure 6. Full simulations (left part) and simulations performed with the MSM using a SBS PhS (right part) are compared to reference measurements. Simulations are represented by the dots and measurements by the lines with small squares. For visualization purpose, curves were rescaled. Depth dose comparisons are presented on the top, the highest curve (normalized to 1) corresponding to the largest field size $\left(30 \times 30 \mathrm{~cm}^{2}\right)$, the lowest curve (normalized to 0.7$)$ corresponding to the smallest field size $\left(5 \times 5 \mathrm{~cm}^{2}\right)$. In-plane profiles at $10 \mathrm{~cm}$ depth for all field sizes $\left(5 \times 5 \mathrm{~cm}^{2}, 10\right.$ $\times 10 \mathrm{~cm}^{2}, 20 \times 20 \mathrm{~cm}^{2}$ and $30 \times 30 \mathrm{~cm}^{2}$ ) are presented on the middle. In-plane profiles for a 10 $\times 10 \mathrm{~cm}^{2}$ radiation field, for all depths $(1.5,5$ and $10 \mathrm{~cm})$ are presented on the bottom, the highest curve (normalized to 1$)$ corresponding to the larger depth $(10 \mathrm{~cm})$.

suited to evaluate the overall simulation and which was $2.5 \%$ in this case. We also evaluated the percentage of points passing the gamma comparison using the clinical $3 \% / 3 \mathrm{~mm}$ criterion. All curves had more than $90 \%$ of the point passing the gamma comparison. This comparison has to be interpreted carefully, since it depends strongly on the number of points evaluated, for instance, for a profile of a $5 \times 5 \mathrm{~cm}^{2}$ radiation field, if only one point out of the 12 evaluated does not pass the gamma comparison, the result drops down to $92 \%$. The gamma comparison allowed for balancing the results obtained when analyzing only the dose differences, giving better results for small fields.

Simulations performed with the MSM using the SBS PhS lie within $0.5 \%$ of simulations performed using the reference $\mathrm{PhS}$, using equations (1) and (2). When compared to full simulations, MSM simulations using a SBS PhS lie within $1 \%$ for all curves, except for the profile at $1.5 \mathrm{~cm}$ for the $5 \times 5 \mathrm{~cm}^{2}$ radiation field, where $\epsilon_{p}=2.2 \%$ and $\epsilon_{n}=1.1 \%$. Looking at the gamma comparison between full simulations and measurements, all curves passed the comparison with a score of $100 \%$ except the PDD of the $5 \times 5 \mathrm{~cm}^{2}$ field, the cross-plane 
profiles at $1.5 \mathrm{~cm}$ and $10 \mathrm{~cm}$ for the $20 \times 20 \mathrm{~cm}^{2}$ field and the in-plane profile at $10 \mathrm{~cm}$ for the $30 \times 30 \mathrm{~cm}^{2}$ field for which the number of points passing the gamma comparison were $97.5 \%, 98 \%, 96 \%$ and $99 \%$, respectively.

The results presented above suggest that no significant bias was introduced by the SBS tool and that the latter can be used safely. Thanks to the full simulations, we evaluated the bias introduced by the MSM. It seems that the MSM introduced a small bump in the profile centers (figure 6), which is even more significant for small fields and might explain the largest discrepancy observed for the $5 \times 5 \mathrm{~cm}^{2}$ radiation field when compared to full simulations. This artifact might be due either by other photon sources not taken into account in this model, or by the binning resolution currently used. It was pointed in section 2.7 that the direction histograms in the center of the PhS suffer of higher statistical uncertainties. Simulations performed by reading the reference $\mathrm{PhS}$ did not show up a similar bump in the profile centers, suggesting that the artifact observed is most likely due to the binning resolution. This result was emphasized by the gamma comparison, which showed a better agreement for full simulations. When compared to full simulations and measurements, the overall agreement obtained using the MSM is encouraging, even if deeper investigations are required to remove the artifact introduced in the profile centers. Part of the results obtained with the MSM using the SBS $\mathrm{PhS}$ and for full simulations are presented in figure 6.

\section{Conclusion}

The goal of this study was to investigate the potential of the new GATE v6.0 release for radiation therapy simulations and the description of a general methodology for Linac simulations. We assessed the advanced DoseActor tool, which allows for energy, dose and statistical uncertainty scoring, the PhS capabilities and the SBS variance reduction techniques combined with filters. Additionally, a MSM was proposed in order to model the patient-independent fluence before the secondary collimator. The possibility of using GATE on the EGEE grid was also presented.

We simulated a 6 MV Elekta Precise Linac photon beam and compared depth doses, in-plane and cross-plane profiles with reference measurements in water for several field sizes ranging from $5 \times 5$ to $30 \times 30 \mathrm{~cm}^{2}$. Phase space files of $1.8 \mathrm{~GB}$ stored above the secondary collimator (patient-independent section) were converted into $5 \mathrm{MB}$ source description files (5404 histograms). The SBS tool was found to increase the photon output rate by a factor 9.7, while the efficiency of a full simulation from the electron beam to the water phantom was increased by a factor 6.2. The proposed MSM derived from the PhS was used as input to simulate the photon beam interactions in the patient-dependent section and to compute the dose distributions in the water phantom.

Our investigations suggested that the SBS tool can be safely used without biasing the simulations. Full simulations performed on the EGEE Grid and $\mathrm{PhS}$ reading allowed for evaluating the MSM. Simulations using the MSM was found to agree within $1 \%$ of full simulations, except for the $5 \times 5 \mathrm{~cm}^{2}$ radiation field, for which errors up to $2.2 \%$ arose. It is noteworthy that the MSM introduced a small bump into the profile centers, which is more significant for small fields and might explain this largest discrepancy. Gamma comparisons were performed: the number of points passing the $3 \% / 3 \mathrm{~mm}$ clinical criterion, when compared to the whole set of measurements, was always above $90 \%$ and $95 \%$, for MSM and full simulations, respectively.

This preliminary study demonstrates that GATE can be used for radiation therapy applications. Its simple macro file structure significantly facilitates the elaboration of GEANT4 simulations. Advanced validation studies with different energies, non-squared fields and multileaf collimator will be performed in a subsequent and detailed validation phase. Absolute 
dosimetry comparisons will be also necessary in order to fully evaluate the accuracy of the platform and investigate systematic errors. Further comparisons with other well-validated MC codes like EGSnrc/BEAMnrc are also contemplated. Because it is based on GEANT4, the GATE platform allows for simulating conventional electron and photon beams, as well as proton, carbon and other ion beams for hadron therapy, thus opening interesting perspectives for the use of GATE as a medical physics-oriented MC platform.

\section{Acknowledgments}

Our work was funded by the French National Research Agency: fGATE project (ANR-06CIS-004).

\section{References}

Agostinelli S et al 2003 Geant4-a simulation toolkit Nucl. Instrum. Methods A 506 250-303

Ali E S M and Rogers D W O 2007 Efficiency improvements of x-ray simulations in EGSnrc user-codes using bremsstrahlung cross-section enhancement (BCSE) Med. Phys. 34 2143-54

Allison J et al 2006 Geant4 developments and applications IEEE Trans. Nucl. Sci. 53 270-8

Apostolakis J et al 2009 Geometry and physics of the Geant4 toolkit for high and medium energy applications Workshop on Use of Monte Carlo Techniques for Design and Analysis of Radiation Detectors, Radiat. Phys. Chem. 78 859-73

Brunner F C, Khoury R, Benoit D, Meessen C, Bonissent A and Morel C 2009 Simulation of pixscan, a photon counting micro-CT for small animal imaging J. Instrum. 4 P05012

Camarasu-Pop S, Glatard T, Mościcki J T, Benoit-Cattin H and Sarrut D 2010 Dynamic partitioning of GATE Monte Carlo simulations on EGEE J. Grid Comput. 8 241-59

Carrier J F, Archambault L and Beaulieu L 2004 Validation of GEANT4, an object-oriented Monte Carlo toolkit, for simulations in medical physics Med. Phys. 31 484-92

Chetty I J et al 2007 Report of the AAPM Task Group no. 105: issues associated with clinical implementation of Monte Carlo-based photon and electron external beam treatment planning Med. Phys. 34 4818-53

Chetty I J, Rosu M, Kessler M L, Fraass B A, Haken R K T, Kong F-M S and McShan D L 2006 Reporting and analyzing statistical uncertainties in Monte Carlo-based treatment planning Int. J. Radiat. Oncol. Biol. Phys. 65 1249-59

Cho S H, Vassiliev O N, Lee S, Liu H H, Ibbott G S and Mohan R 2005 Reference photon dosimetry data and reference phase space data for the $6 \mathrm{MV}$ photon beam from varian clinac 2100 series linear accelerators Med. Phys. 32 137-48

De Beenhouwer J, Staelens S, Vandenberghe S, Verhaeghe J, Van Holen R, Rault E and Lemahieu I 2009 Physics process level discrimination of detections for GATE: assessment of contamination in SPECT and spurious activity in PET Med. Phys. 36 1053-60

Deng J, Jiang S B, Kapur A, Li J, Pawlicki T and Ma C M 2000 Photon beam characterization and modelling for Monte Carlo treatment planning Phys. Med. Biol. 45 411-27

Faddegon B A, Asai M, Perl J, Ross C, Sempau J, Tinslay J and Salvat F 2008 Benchmarking of Monte Carlo simulation of bremsstrahlung from thick targets at radiotherapy energies Med. Phys. 35 4308-17

Foppiano F, Mascialino B, Pia M G and Piergentili M 2005 Geant4 simulation of an accelerator head for intensity modulated radiotherapy Proc. of The Monte Carlo Method: Versatility Unbounded in a Dynamic Computing World (Chattanooga, TN, 17-21 April 2005) (LaGrange Park, IL: American Nuclear Society) CD-ROM

Frisson T, Zahra N, Lautesse P and Sarrut D 2009 Monte Carlo based prediction of radiochromic film response for hadron therapy dosimetry Nucl. Instrum. Methods Phys. Res A 606 749-54

Fix M K 2001 A multiple source model for 6 MV photon beam dose calculations using Monte Carlo Phys. Med. Biol. 46 1407-27

Fix M K, Keall P J and Siebers J V 2005 Photon-beam subsource sensitivity to the initial electron-beam parameters Med. Phys. 32 1164-75

Fix M K, Keall P J, Dawson Kathryn and Siebers Jeffrey V 2004 Monte Carlo source model for photon beam radiotherapy: photon source characteristics Med. Phys. 31 3106-21

Fix M K, Keller H, Ruegsegger P and Born E J 2000 Simple beam models for Monte Carlo photon beam dose calculations in radiotherapy Med. Phys. 27 2739-47 